Copyright (C) 2021 by Cherkas Global University

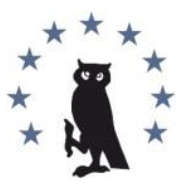

Published in the the USA

International Journal of Media and Information Literacy

Has been issued since 2016.

E-ISSN: $2500-106 \mathrm{X}$

2021. 6(2): 436-446

DOI: 10.13187/ijmil.2021.2.436

https://ijmil.cherkasgu.press

\title{
Virtual Communities as Sites of Market Genesis: A Netnographic Study of Netflix India and Amazon Prime Video India's Facebook Groups
}

\author{
Sonali Srivastav a, b, Shikha Rai a, * \\ a Indira Gandhi National Open University, New Delhi, India \\ ${ }^{\mathrm{b}}$ National Institute of Fashion Technology, Bhubaneshwar, Orissa, India
}

\begin{abstract}
The study is aimed at examining the online brand image building and user engagement strategies adopted by the two Video on Demand Service providers, Amazon Prime Video India and Netflix India. It also analyses the communication patterns between the subscribers and the brand. The paper utilizes a method called Netnography or virtual Ethnography and studies two communities - the official Facebook groups - Netflix India and Amazon Prime Video India. Data was collated by acquiring two approaches, Observation and Extraction of archival data. The method not only shed light on strategies used by the service providers to engage the audience, but also witnessed the efforts by the brands of converting consumers into fan bases. It helped in discerning the communication process within the community, within the members and with the brand, contributing towards a brand building exercise on the Facebook group. Lastly, the data gathered also helped in evaluating quantitatively and qualitatively if the methods are working in favor of the brands or not. The results are not only indicative of how social media presence affects a brand image, but also how fan cultures have replaced the relationship of a service provider and buyer. These groups are emerging not only as sites of creating market space, but also for fan congregations. Participants interact not only with the brand, but also with each other and further inviting new subscribers to the group as well, laying a case for virtual word of mouth publicity. These groups are also instrumental in providing a quick feedback to the administrators of the page about the strategies adopted for marketing their content through Facebook groups.
\end{abstract}

Keywords: Facebook, Netflix, Amazon, virtual communities, netnography.

\section{Introduction}

Video on Demand services in India have created more than a foothold for themselves in past few years. Providing on demand entertainment, on the move, as per consumer's taste, these services are also hosting content from TV and Films apart from producing originals for the web platforms. Following the subscription-based or ad-based models, these services use streaming to reach its viewers, thus also called SVoD services. By the end of 2018, an estimated 30 million subscribers were hooked on to various SVoD platforms. The industry revenue estimation is around 17 billion by the beginning of 2019. Attracting a subscriber base mainly in the age group 25- 34 years, most of its viewers lie in middle - high income group (Statista, 2019).

Netflix India was launched in January 2017, and its global annual revenue was estimated to be 85 billion in 2017. Still using TV as a medium of play, 70 percent of the Netflix users watch it on TV (Kafka, 2019). Netflix also spends more than any other SVoD platform to produce own content.

\footnotetext{
${ }^{*}$ Corresponding author

E-mail addresses: srivastav.sonali.89@gmail.com (S. Srivastav), shikharai@ignou.ac.in (S. Rai)
} 
Users also prefer watching Netflix's original content more than the acquired content, citing its algorithm of suggestion as its USP. Releasing its first Indian original web-series in July 2018, Sacred Games, Netflix is targeting a global market with the content, by releasing it in Brazilian, Spanish and Italian dubbing as well, along with Hindi and English (Kafka, 2019). While India is not the largest revenue generating market for Netflix yet, it is growing at a rapid rate (Chauhan, 2019; Toms, 2019).

Amazon Prime Video is a second most popular SVoD service provider in the world (Wallach, 2021). Belonging to one of the largest e-commerce companies in the world, Amazon Prime Video is backed by a huge conglomerate. Since its launch in India in late 2016, it has been gaining rapid market share. Also using TV as a primary medium of display, Amazon's USP according to its users is a large variety of content. In India, while Amazon started with Breathe in 2018 as its first title, it has been acquiring regional content as well, thus tapping into a regional audience base as well (David, 2019).

In times of today, Internet marketing has emerged as a tool which utilizes the networked digital technologies to market a brand. Social media is appearing as an extension and often a replacement of real-world interaction. Using social media as a platform to market themselves, these SVoD service providers connect with an audience base present online $24^{*} 7$ (Horbel et al, 2016). Social media helps in creating avenues for not only existing customers but also for people who might get interested enough to be converted into a selling point. These may identify themselves as users, subscribers or fans of the service providers (Mann, 2016).

While the demographic profile of their target base is metropolitan, net savvy and English speaking, the SVoD service providers need to create further different market segments for themselves to thrive. Amongst the lot of existing seven-eight SVoD service providers in India, Amazon Prime Video India and Netflix India have emerged as platforms with the largest subscriber base. But both of these brands have different marketing strategies and adopt different methods to reach out to their audience bases. The current research in question ventures into the online image building strategies adopted and practiced by these two brands in India, specifically on Facebook. The study also looks at various audience engagement methods and patterns emerging in the Facebook groups of these two SVoD service providers.

\section{Materials and methods}

Aim: This paper is aimed at studying the online brand image building strategies and audience engagement patterns adopted by two SVoD Service providers in India.

Objectives: The specific objectives of this study are as follows:

1. To ascertain the audience engagement strategies adopted by Netflix India and Amazon

Prime Video India through their Facebook Groups.

2. To evaluate how their Brand Image is created, established and propagated through these groups.

3. To assess how does audience interact with the brand, the content and with each other in these communities. study:

Research Questions: Following are the research questions to be answered by the end of the

1. What are the engagement activities taken up by Netflix India and Amazon Prime Video India for their subscriber base on their official Facebook groups?

2. How do their engagement approaches reflect on their marketing strategies and generating a brand image?

3. How does the audience respond towards these engagement approaches?

4. What are the patterns of communication within the Facebook communities of Netflix India and Amazon Prime Video India?

Method: Drawing parallels from field based ethnographic studies where the researcher studies the behavior of communities in the real world, this paper utilizes a method called Netnography or virtual ethnography to study the communication patterns in virtual groups (Kozinets, 2015).

The concept of user engagement is still a novel one in the Indian Markets, and utilization of Social Media tools provided by any platform, in this case Facebook, has not been studied extensively. Apart from this, the communities that emerge online, following, discussing and promoting a sort of content also need to be studied for emerging patterns of behavior and motivations. 
Facebook provides a platform to create groups which can add billions of people at one go. Moderated by one person, these groups have content posted by the administrator, while other participants or subscribers of the group react and interact with it. Reactions are generally in form of emoticons and comments. Interaction is when subscribers of the groups tag each other, strike up a conversation with the administrator or each other through comment threads on the posts.

Sample: Two Facebook groups, Netflix India and Amazon Prime Video India, run officially. Netflix had 4,78,92,712 members and Amazon Prime Video India had 16,98,328 members (extracted on 1 July 2018). During the period of study Netflix India posted 122 posts and Amazon Prime Video India posted 283 posts on their respective Facebook groups. These posts by the administrator and the reactions and comments generated on each by the subscribers were studied.

Time period: 1 July 2018 - 7 October 2018 (14 weeks)

Tool: Participatory Observation method was used to collect data. The researchers joined these two Facebook groups and studied the posts, the reactions on them and the meta reaction by the administrators of the page.

The data was collected by two approaches:

a) Observation of activities

The researcher became a participant in the virtual community and spent an hour on. Daily basis on each of these platforms. She read the posts, analyzed their purpose (to launch new content, to recall old content etc.), assessed their content (tries to involve humor, tries to be informatory, entertaining etc.), studied the comments (if the group subscribers are striking up a conversation, or they are only reacting) and estimated the overall reaction towards the post. She also scrutinized the conversation/s taking place between the page administrators and subscribers and subscribers with each other. All these observations made for the field notes, albeit taken down in an excel sheet for the ease of use. This approach helped in collecting qualitative data from the sample.

\section{b) Extraction of archival data}

The data pertaining to number of posts, various kinds of reaction on them and their numbers, counter comments, general purpose and type of text of the posts and the content, which is being posted about, was collected by the method of manual extraction. The posts were extracted after 3 weeks of their posting so that the reactions they had to generate would have been accumulated (Kozinets, 2015). While various data scraping tools are available in the market, this process helped in gathering data more organically and giving time to observe the post for longer. This data was saved in excel sheets in order to make comparison and comprehension simpler and easier. This approach also helped in collecting quantitative data from the sample.

Scope of the Study: The study is limited to Indian markets and thus focusses only on the Facebook groups created specifically to reach out to the Indian audience base.

\section{Discussion}

An extensive review of literature was done before embarking on the study. Following research were found to be touching upon the topic directly and tangentially.

Focusing on streaming as an emerging video entertainment industry, Burroughs claimed that it was rapidly replacing television by introducing a phenomenon called 'cord-cutting', which referred to the act of removing the device connected by a cord, i.e., the TV and going cordless, like in case of laptops and mobile phones. He called the initial Netflix audience base as the 'Cord Cutters'. He also compared Netflix's policies with Blockbuster's, which was once its prime competitor. Blockbuster did not adapt the streaming model and stuck with DVD rentals, thus bringing a premature demise to the competing company. Then moving to targeting TV's audience base, Netflix adapted to subscription-based Video-on-Demand (VOD) model. Then it shifted to cutting into HBO's model by producing original content and releasing it. Thus, it branched out vertically and horizontally by approaching segments after segments to expand itself (Burroughs, 2015; 2018).

DeCarvalho et al. in their case study of Netflix list conducted a SWOT (Strengths, Weaknesses, Opportunities, Threats) analysis of the company in 2014, in American context, based on the public literature about the company. Few strengths according to their analysis of the company were: it had become a well-established name with an expanding user base, could boast of a well-versed management team and had a sharp suggestion algorithm. It was foraying into newer 
markets and formulating the strategies based on the specific culture. For e.g., Use of Food trucks in Mexico for running their advertising campaign (DeCarvalho et al, 2018).

Amongst many of the platforms facilitating personal, group and mass interaction is Facebook. With the help of one of its features, Facebook groups, a number of online communities have cropped up in past decade (Ahuja et al., 2018). While discussing Facebook's features that enable marketers to gather, save and use Big Data analytics to market their products, Gosling et al deliberate on the psychological motivations on the part of users, or group subscribers that incentivizes the use of the platform for them. As psychologists, they also provide a set of guidelines to utilize the features of the medium to its best potential for the marketers (Gosling et al, 2015).

These groups create a virtual alcove for hosting conversation and engagement based on a theme or purpose. Ranging from facilitating e-commerce to organizing fan forums to sharing jokes and memes, these groups have an administrator who moderates the conversations and content (Lahav et al., 2018). These groups may have members ranging from five to few millions. Armed with features like tagging, sharing, hash-tagging and memes, the marketing strategies have evolved many a fold over the social network (Demirhan et al., 2017).

The official Facebook pages run by these service providers, are one of the podiums utilized for the same purpose. They are also utilized to create a fan base and to promote and extend engagement with their content. Numbered in millions, these groups can be joined by anyone, subscriber of the service or not (Dahl, 2018).

Aiello et al in their work traced the users of few luxury brands who had subscribed to their respective Facebook groups as well. According to their study, the consumers felt more connected with the brands and thus showed greater brand preference and loyalty. The researchers also shortlisted five factors which lead to Social Media Marketing plan designing: entertainment, interaction, trendiness, customization and word of mouth. The study concluded that Social Media marketing created a considerable impact on brand equity of these luxury brands (Aiello et al, 2016).

Emrich et al discuss the benefits of liking and following a group on a networked platform like Facebook. They evaluate that the friends of the subscribers also view a brand more positively when they see people in their friend list joining these groups and interacting with the content. They concluded that the word of mouth still has to supplement the 'word of web' in order to create another consumer (Emrich et al, 2017).

Kozinets et al. elaborate upon the virtual liking of a Facebook page and brand evaluation by consumers. With the help of an online experiment with pre and post measurements of group activity, the researchers found a significant positive increase in brand evaluation by the followers of a group, compared to that of non-followers. The study highlighted the importance of engagement through interactivity on social media platforms (Kozinets et al., 2018).

Dwivedi et al. debate on three types of groups existing on Facebook, Associative, Aspirational and Advertising. The research threw light on the fact that users of Facebook join these groups with different motivations while marketers try to mould those motivations into creating selling points. While advertising oriented groups had the least engagement, it was the associative type of groups that led to most credible exchange between brand and consumers (Dwivedi, 2019). Kapoulas et al. agree with Facebook communities emerging as sites for marketing the brands. The researchers also used netnography as a tool to study the Fashion industry in Southeast Europe. (Kapoulas et al., 2020)

Buschow et al., worked on differentiating between the brand perception of Netflix and Amazon Prime video in the Video on Demand Market in Germany and found their strategies to be very different from each other. While Netflix was targeting the younger population, Amazon was targeting the mass. (Buschow et al, 2020)

While the works employed online experiments, surveys and meta-analysis, neither of them utilized Netnography as a method of study (Bridges, 2016). Also, Netflix and Amazon in India are relatively new in the market and are Internet based service providers. Thus, it can safely be assumed that these companies would be able to utilize the features of a social networking platform to market themselves better. Thus, to absolve these gaps, the study in question was designed.

\section{Results}

After collecting the data for a period of 14 weeks, through two approaches, observation and extraction, few findings emerged. Data extracted from the page, in form of user statistics, number 
of posts, comments and reactions is presented in tabular format in Table 1. Data from observation, which is qualitative in nature, is further discussed along with examples.

Netflix India's official Facebook group had 5,23,63,660 people subscribed to the page as of November 2018. The page sees about 1-2 posts every day engaging with its subscribers through text, videos, memes, pictures and games. During the period of the study, 7 new titles were added by Netflix out of which 4 were Indian. The fan base increased at the rate of about 2 lakhs new subscribers every week.

Amazon Prime Video India's official Facebook page had 44,13,384 subscribers and followers as of in November 2018. During the period of study, the page hosted about 3 posts on an average every day. The subscribers grew in numbers at a steady rate, that is about 15,000 new subscribers every week, but saw an immense growth in two weeks, i.e., 16 September to 30 September again succumbing to the initial rate of growth.

Table 1. Comparative reactions of both groups

\begin{tabular}{|l|l|l|}
\hline \multicolumn{1}{|c|}{ Elements of study } & Netflix & Amazon \\
\hline Posts by the group administrator & 122 posts & 283 posts \\
\hline Average posts per day & 1.33 posts & 3.07 posts \\
\hline Reactions by the subscribers & \multicolumn{1}{|l|}{} \\
\hline Like & 452171 & 2178382 \\
\hline Average Likes per post & 3706.32 & 7697.46 \\
\hline Love & 41205 & 46941 \\
\hline Love (per post) & 337.75 & 165.9 \\
\hline Laugh & 38732 & 22368 \\
\hline Laugh (per post) & 317.48 & 79.04 \\
\hline Wow & 7975 & 7159 \\
\hline Wow (per post) & 65.37 & 25.30 \\
\hline Angry & 0 & 156 \\
\hline Angry (per post) & 0 & 0.55 \\
\hline Sad & 19 & 184 \\
\hline Sad (per post) & 0.16 & 0.7 \\
\hline Shares & 50880 & 44664 \\
\hline Shares (per post) & 417.05 & 157.82 \\
\hline Comments & 46745 & 27721 \\
\hline Comments (per post) & 383.16 & 97.95 \\
\hline Interaction by administrator & \multicolumn{2}{|l|}{} \\
\hline Replies to comments by administrator & 492 & 1045 \\
\hline $\begin{array}{l}\text { Replies to comments by administrator } \\
\text { (per post) }\end{array}$ & 4.03 & 3.69 \\
\hline Page statistics & $4,78,92,712$ & $16,98,328$ \\
\hline No of subscribers on July 1, 2018 & $5,08,69,395$ & $42,78,213$ \\
\hline No. of subscribers on October 7, 2018 & $29,76,683$ & $25,79,885$ \\
\hline Growth & $6.22 \%$ & $151.90 \%$ \\
\hline Rate of growth during the period of study & \multicolumn{2}{|l|}{} \\
\hline
\end{tabular}

\section{Netflix}

Netflix posted 122 posts in the period of 14 weeks of study. On an average, each post was liked by its subscribers 3706.32 times, loved 337.75 times, laughed at 317.48 times, wowed 65.36, angered o times and saddened 0.16 times. Each post was shared by its subscribers 417.05 times on an average and generated 383.16 comments. The administrator responded back to the comments 4.03 times per post.

Posts: Netflix's posts are humorous in nature, at times joking about their own content/actors through jokes, GIFs or videos as posts. For example, a popular GIF from the series Sacred Games (2018) with one of the lead actors aimed at the subscriber base who wanted the week to end on 
Friday so that they can go home and catch Netflix. Through posts like this, Netflix: a) Advertises its content; b) Typecasts its audience base as the ones working in Multinational corporate setups who typically work till 7 and have weekends off, while accepting to be a part of it; c) Brands itself as a leisure activity to be done on weekends.

The comments or retorts by the group members on such posts are also similar in nature. This light-hearted banter gets people attached with the group and posts informally. When people do not get the inside jokes, Netflix urges them to watch certain content to 'join in the group'.

In a post on September 18, Netflix India commented on its own post related to Sacred Games (2018), "25 din mein kya hoga? Trivedi kyo bachega? What is a tabut? So many unanswered questions! (roughly translated as - What will happen in 25 days, stay with us to know)".

It was also found that the audiences wanted to belong to the group and did not want to miss out on the interaction. For example, the audience interaction in the comments showed one subscriber asking the other one to watch certain series so that she can also understand the memes. People were found asking each other to watch a particular show to catch up or make plans to watch stuff together, thus making the interaction transcend into the real world uninterrupted. While this makes for a word-of-mouth publicity in the virtual world, the group also witnesses people hinging their personal relationships to particular shows/content.

It was also observed that hyperlinking and tagging of profiles on posts created an easy flow of traffic leading to better publicity of brands through Facebook pages. People asked each other to buy the subscription, doing the hard selling for the brand by themselves. Groups like this, made every individual a promoter, and Netflix understands that apart from creating entertaining content, if they manage to create entertaining and engaging posts, they would ensure a class of audience engaged with them. This phenomenon helps Netflix by:

a) Creating a word-of-mouth publicity in the virtual world;

b) Banks on FOMO (fear of missing out);

c) Creates an important piece in the fabric of pop culture;

d) Gets more subscribers.

Subscribers were found to complain about having to wait too long, while Netflix responded back with a joke. It seemed to be common consensus within the group members that binge watching was addictive, yet they were hooked to it. The content itself was very dark, but Netflix managed to sell it with lighthearted jibes. Adapting the meme and GIF culture on the Internet, Netflix was found to indulge in self-deprecating humor popular with the youth on platforms like this.

Netflix also used memes and GIFS from its own shows to answer back to comments. For e.g., on an appreciative comment on a post about Orange is the New Black (2016) will receive a reply in form of a GIF from the Stranger Things (2016). There were also instances where Netflix was found to be reminding the audience of its older products through its posts. For example, there was a post about the series Little Things (2018) and the GIF as the reply was from Stranger Things (2016). By doing this, Netflix:

1) Advertises its old content;

2) Creates a visual text for better engagement;

3) Is able to connect with its subscribers.

When not discussing its own content, the jokes would draw upon other popular culture references. For example, the researchers studied the responses by Netflix referring to Shaktiman (Hindi TV Show 1997-2005), while another referred to Harry Potter (Books (1997-2007), films (2001-11). By doing this:

a) Netflix created relatable content for its millennial subscribers;

b) It carved a niche for its audience base.

Apart from producing memes and GIFs from earlier shows, Netflix was also found to be producing special video ads and posters to advertise its content. For example, an ad for a perfume named Ghoul, to advertise Ghoul (2018), talk shows and a post containing popular actress Kareena Kapoor running behind the train from Jab We Met (2007) compared with another clip of actor Saif Ali Khan running behind a train in Sacred Games (2018) in split windows.

The posts were also found to be celebrating friendship day, romance day, relationships and Indian Supreme court's judgement on Section 377, a section which discusses homosexuality. These posts contained a supporting montage from all its shows, original and acquired. Having a grip on the nerve of the socio-political scenario of the country, the posts also had news footage to advertise 
their own content. For example, there were clips from religious leader Maa Anand Sheela's controversial speeches, used to advertise content based on similar ideas.

Few posts were also found to focus on people who created or contributed to creation of content. Netflix created heroes out of actors Radhika Apte, Siddiqui and writer George RR Martin, referring to them in various posts. For example, the researchers found a few posts that referred to watching pirated content and Netflix responded with a gif of Nawazuddin Siddiqui loading a gun. The text said, 'Kya bola bay' (translation: what did you say?). Netflix also created an extended persona for the characters from its content while creating these posts. For e.g., the posts referred to characters by their reel and real names, for e.g., Nawazuddin would be Gaitonde (a character from Sacred Games (2018)) or vice versa, blurring the lines between the real and the virtual personalities.

It was observed that content in Hindi was found to have greater engagement than the content in English, Netflix had a mix of both the languages and pop culture references while creating the posts. Group members very specifically celebrated 'anti Ekta Kapoor content' (a specific category of TV soap operas in India that are highlighted by melodrama and overly display of emotions) and publicly bashed other group members who dissented. Many social media pages run by media houses like VH1, ScoopWhoop, MAD by Rob etc were also found to be engaging with posts. This further helped by:

a) Getting the fans from both the pages to increase the traffic;

b) Collaborating with lesser-known celebrities on the internet to promote their work and in return tap into a niche audience base;

c) Promoting alternative icons through the posts and content.

Meta Reaction: Netflix page was found to be run by an administrator, who responded back to first few comments made on every post, albeit with a funny turn. It responded to threats of unsubscribing, pirating, but shied away from addressing posts about competitors or any other sort of content. It was also found to respond to the subscriber's requests to add new content. Responses were also found for messages sent through Facebook messenger almost instantly, as per the feedback by the subscribers of the group. Group members, who were also present on other platforms, redirected traffic to the Netflix app and Facebook groups as well. While on one hand this pointed at a loyal fan base and social media promoting cross brand promotion, it also hinted at a consumer base who wanted to watch certain content, wherever it may be hosted.

\section{Amazon Prime Video India}

In the period of 14 weeks, the page saw a total of 283 posts, amounting to a little more than 3 posts every day. Each post was liked on average about 7697 times, loved 166 times, laughed at by 79 subscribers, wowed 25 times, angered 0.55 times and saddened 0.7 subscribers. Each post was shared 158 times and commented upon 98 times with administrator responding back 3.7 times per post.

Posts: Amazon is an established gigantic ecommerce brand with Amazon Prime being a premium membership to get benefits of many kinds on ecommerce website. Prime Video is an extension of the same, providing content through online platforms. Thus, the platform has its own web commerce page to start advertising from. The posts also provided links to webpages of Amazon, Amazon Prime Video and IMDB as well, creating a mesh of networked websites. It was also found to utilize the page to sell its other content/platforms, such as Prime Reading.

Prime has emerged as the lead and most frequent acquirer of content from Hollywood and Indian Cinemas in recent times. Thus, the posts also advertise recently acquired titles. The page changes its cover photo frequently based on recent acquirements. Appealing to regional fan bases, Prime acquires popular content in Tamil and Telugu as well and not just in Hindi. It also releases international titles dubbed in 12 local languages ranging from Bengali to Kannada. Apart from acquiring new released titles, Prime also acquires old titles such as Malgudi Days (1987) as well, thus providing a wider content base to its subscribers.

Balancing the act between original and acquired content, while the administrator posts greater number of posts on original content (168/283 posts), it is the posts relating to acquired content that see greater audience engagement.

The posts range from celeb endorsements of few shows, Birthday wishes for various Hollywood or Indian film celebrities, snippets from its original Stand-Up Comedy Show Comicstaan (2018), collaborations with Internet Celebs and small production houses to incorporate a wider content base. The posts also had tags for celebrities to: 
a) Widen the audience base and invite more traffic from the fan bases;

b) Give a chance to the subscribers to interact with the celebrities on one to one basis;

c) Create a virtual presence of these celebrities on their page.

The posts tagged the film celebrities such as Chris Pratt and Shilpa Shetty to known faces on Internet such as stand-up comedians Sorabh Pant, Tanmay Bhatt and Biswa Kalyan Rath. There were few collaborative and outsourced videos from other media houses such as Arre, Filter Copy and Kavithalaya, banking on the internet friendly content these sites create.

Few posts created were also jokes and memes created on popular social media trends such as Kiki challenge and PUBG, the trending game. This was done in order to: 1) Attract the young audience base who is familiar with these phenomenon; 2) Use hashtags with these videos to make their content and thus their page indexed and easily locatable.

Reactions: While emerging as the second most populous group in the sample, it saw lesser tagging by subscribers. This could be due to two reasons: 1) Prime advertises its content on few TV channels as well, thus making up for less word-of-mouth advertising; 2) It has established itself as a family brand, to be seen on TV set with the help of Amazon Fire Stick, thus including an audience base with a wider age bracket.

Most of the comments were not related to content already posted but requesting for more titles to be added. Thus, subscribers did not treat the posts as points of interaction and feedback, but particularly as request boxes. Most reactions were garnered by the posts wishing birthday to various celebrities. Subscribers who were fans wrote comments on the post wishing birthday to their favorite celebrities. Apart from these posts, regional content saw more interaction as compared to Hindi or English Content.

Meta Reaction: As per the observations and feedback, the administrator responds to personal messages sent through messenger almost immediately. The page administrator/s react to the posts in a manner more suited to Customer Service personnel. They responded to compliments, complaints and requests and forwarded their queries to relevant departments.

The staff recruited for responding on behalf of Amazon display their names at the end of the posts, so that: 1) In case the subscriber wants to reach out further, they know who has been their point of contact; 2) Amazon does not have to bear the responsibility of these responses as the brunt lies on the shoulders of the person who is named; 3) To invoke a feeling of a customer care cell or a corporate set up.

\section{Conclusion}

With the help of the data collected - quantitative through archives and qualitative through observations, few factors emerged organically. The content posted on the platforms, audience's reaction to this content, further engagement by tagging other subscribers or eliciting responses by the administrators of the page highlighted the nature of the communication strategies, while the numbers, in form of subscriber base, number of posts, comments and reactions underlined the success, or failure of these strategies. Further section deals with discussing them one by one for each brand.

Netflix brands itself as a young, cosmopolitan, trending brand which provides content for entertainment. While focusing on creating class content, it also focusses on creating material for posting on the Facebook group, whether it be talk shows, or a montage put together from its shows. The posts were analyzed to chalk out Netflix's existing communication strategies. These include:

1) Humor - Netflix indulges in self-deprecating humor, while creating jokes on self and subscribers as posts and comments. People mostly react to the posts in a positive manner, laugh and love emoticons used much more than angered or sad.

2) Memes - Utilizing humor and GIFs from its own earlier content. This strategy helps twofold; in advertising old content and hooking old audience to new content. This also makes the communication visual, diverging a bit from the usual textual format, harnessing on the collective memory and understanding of the entire subscriber base.

3) FOMO (Fear of missing out) - Netflix drives on the culture propagated by social media, which induces fear of missing out in people. It keeps on reminding subscribers to watch their content, and they in return involve their friends and followers through tagging or hyperlinking.

4) Using pop culture references - This draws upon from the common psyche of the subscribers. Popular characters such as Shaktimaan and Harry Potter are referenced to start up conversation from posts and comments. The posts also refer to celebrities, big or small. This helps 
in tapping into varied fan bases and makes the content reach out to various niches.

5) Responding - while compliments and criticisms are responded back to, the administrator does not revert to threats to give up subscription. It also addresses piracy with jokes and memes.

While these factors ran common through majority of the posts, they also created a window into the marketing strategies as followed by the brand. Few pointers from which are listed below:

1. Netflix banks on its content to do most of the selling. After the series is a month or few weeks old, the administrator assumes that most of the subscribers would have seen it and starts creating jokes and memes. The need to belong and understand the jokes makes others watch the shows.

2. Humor appeal - however dark the content, the posts are lighthearted in nature, in order to entertain, intrigue and $\log$ in at the end of the day.

3. Targets the metropolitan youth, who is part of corporate culture, stays away from home, does not have a TV set, has money to spend, understands English and is a part of social media.

4. Banks on word-of-mouth publicity. Page followers tag their friends in great numbers and thus the number of subscribers increases every week. Administrator also pitches in the conversation two people are having on a thread to make them feel involved and connected.

5. Collaborations. Netflix collaborates with celebrities big or small, other entertainers, small or big time. These collaborations could be acquiring content, producing something together, or merely using a few visuals to sell content. This again helps in tapping into various segments or audience.

6. Personal contact. Calling people by their first names, indulging in back slapping humor, trying to involve subscribers through quizzes and games, Netflix tries to connect to advertisers on a very individual level, and social media aides them in doing this.

Thus, these were few pointers regarding Netflix's Social Media Communication policies group and Marketing Strategies that emerged out of 14 weeks of observation of the official Facebook. The strategies seemed to be working as the subscriber base increased with a steady rate with multiple engagement strategies in place.

Amazon Prime Video India has branded itself as a family entertainer, replacement for TV and films, while borrowing its content from both of them. It works on a subscription model, thus advertising the USP of advertisement less entertainment. Because of such a large market, Prime is not targeting any particular segment as target market but is trying to serve something for everyone. The posts analyzed threw some light on its communication strategies with its diverse consumer base:

1) The Facebook page runs like a Customer Service forum, with the posts being points of interaction.

2) There were attempts at humor, creating suspense, creating a camaraderie with celebrities throw the posts, thus attempting many things as once akin to TV

3) Images and posters comprised of most of the content, thus making the process of post generation faster and mess free.

4) Original content as talked about more, acquired a little less, ratio being 60:40.

These communication strategies meted out as an important part of its marketing strategy as well along with these:

1) Amazon utilizes its brand name and other webpages to sell its content and converge the traffic

2) Amazon ties up with other content giants such as IMDB or Internet Movie Database to advertise the content is has already acquired.

3) Amazon advertises itself through TV ads as well. Thus, not merely depending on the advertising through Internet.

4) Amazon also acquires channels as well as shows in order to tap into fan bases. It has the largest repertoire of content compared to all other platforms.

While its strategies mark it as another mass entertainer venturing in the field of virtual entertainer and video on demand services, its marketing and communication strategies are less personal, more business-like and remind you of corporate giants who surround us through multiple platforms and channels.

In conclusion, with a clear demarcation in strategies and communication patterns, while Netflix manages to use the Facebook's networked platform in a marked enhanced manner, comparatively, the Indian market is responding better to the corporate approach adopted by 
Amazon. Both approaches are helping them exploit their respective target markets. As SVoD is replacing TV as the medium of entertainment, the fulcrum still resides in the TV set as the medium of play and content for the family still being the need of the hour. Thus, it can be stated that while Netflix creates its mark in the more urbane audience groups, Amazon is steadily matching up with its broad mass appeal.

\section{References}

Ahuja et al., 2019 - Ahuja, V., Alavi, S. (2018). Using Facebook as a digital tool for developing trust amongst consumers using netnography and social media analytics: a study of jet airways. Journal of Relationship Marketing: 1-17.

Aiello et al., 2016 - Aiello, G., Donvito, R., Godey, B., Manthiou, A., Pederzoli, D., Rokka, J., Singh, $R$. (2016). Social media marketing efforts of luxury brands: Influence on brand equity and consumer behaviour. Journal of business research. 69(12): 5833-5841.

Bridges, 2016 - Bridges, N. (2016). Facebook as a netnographic research tool. Global Media Journal: Australian Edition. 10(1).

Burroughs, 2015 - Burroughs, B. (2015). Streaming media: audience and industry shifts in a networked society. The University of Iowa.

Burroughs, 2018 - Burroughs, B. (2018). House of Netflix: Streaming media and digital lore. Popular Communication: 1-17. DOI: 10.1080/15405702.2017.1343948.

Buschow et al, 2020 - Buschow, C., Rahe, V., Schlütz, D. (2020). How users approach novel media products: Brand perception of Netflix and Amazon Prime video as signposts within the German subscription-based video-on-demand market. Journal of Media Business Studies: 1-14.

Chauhan, 2019 - Chauhan, H. (2019). Netflix smartly changes its game plan in India The Motley Fool. [Electronic resource]. URL: https://www.fool.com/investing/2018/o8/20/netflixsmartly-changes-its-game-plan-in-india.aspx

Dahl, 2018 - Dahl, S. (2018). Social media marketing: Theories and applications. Sage.

David, 2019 - David, S. (2019). 'Breathe' Amazon Prime Series Review: R Madhavan Slowly Takes Your Breath Away. [Electronic resource]. URL: https://www.news18.com/ news/movies/ breathe-amazon-prime-series-review-r-madhavan-slowly-takes-your-breath-away-1657285.html

DeCarvalho et al., 2018 - DeCarvalho, L., Martínez-Carrillo, N. (2018). Serving (Fetishized) Time: An Intersectional Analysis of Netflix's Food Trucks in Mexico and the United States. The Journal Of Popular Culture. 51(2): 487-510. DOI: 10.1111/jpcu.12670

Demirhan et al., 2017 - Demirhan, E., Horzum, M.B. (2017). The role of chronotype on Facebook usage aims and attitudes towards Facebook and its features. Computers in Human Behavior. 73: 125-131.

Dwivedi et al., 2019 - Dwivedi, Y.K., Islam, R., Mukerji, B., Rana, N.P., Shareef, M.A. (2019). Social media marketing: Comparative effect of advertisement sources. Journal of Retailing and Consumer Services. 46: 58-69.

Emrich et al., 2017 - Emrich, O., John, L.K., Gupta, S., Norton, M.I. (2017). Does "liking" lead to loving? The impact of joining a brand's social network on marketing outcomes. Journal of Marketing Research. 54(1): 144-155.

Gosling et al., 2015 - Gosling, S.D., Kosinski, M., Matz, S.C., Popov, V., Stillwell, D. (2015). Facebook as a research tool for the social sciences: Opportunities, challenges, ethical considerations, and practical guidelines. American Psychologist. 70(6): 543.

Horbel et al., 2016 - Horbel, C., Popp, B., Wilson, B., Woratschek, H. (2016). Relationship building through Facebook brand pages: The multifaceted roles of identification, satisfaction, and perceived relationship investment. Journal of Strategic Marketing. 24(3-4): 278-294.

Kafka, 2019 - Kafka, P. (2019). Netflix Expands Into India, but Not China. [Electronic resource]. URL: https://www.recode.net/2016/1/6/11588550/netflix-expands-into-india-but-not-china

Kapoulas et al., 2020 - Kapoulas, A., Miaoulis Jr, G., Xharavina, N. (2020). Netnography as a marketing research tool in the fashion industry in Southeast Europe. International Journal of Market Research. 62(4): 499-515.

Kozinets et al., 2018 - Kozinets, R.V., Perren, R. (2018). Lateral exchange markets: How social platforms operate in a networked economy. Journal of Marketing. 82(1): 20-36.

Kozinets, 2015 - Kozinets, R.V. (2015). Netnography. John Wiley \& Sons, Ltd. 
Lahav et al., 2018 - Lahav, T., Roth-Cohen, O. (2018). Going undercover: Online domestic tourism marketing communication in closed and open Facebook groups. Journal of Vacation Marketing: 1356766718796054.

Mann, 2016 - Mann, L.M. (2016). "Come TV with us": the business strategies, discourses, and imagined audiences of Netflix and Hulu. Ph.D. Dis.

Statista, 2019 - "Video-On-Demand - India | Statista Market Forecast". 2019. Statista. [Electronic resource]. URL: https://www.statista.com/outlook/201/119/video-on-demand/india\# market-revenue

Toms, 2019 - Toms, M. (2019). Cometh the hour: six elite battles that will shape India's New Economy in 2019 - ET Prime. [Electronic resource]. URL: https://prime.economictimes. indiatimes.com/news/67358271/technology-startups/cometh-the-hour-six-elite-battles-that-willshape-indias-new-economy-in-2019

Wallach, 2021 - Wallach, O. (2021). Which Streaming Service Has the Most Subscriptions? [Electronic resource]. URL: https://www.visualcapitalist.com/which-streaming-service-has-themost-subscriptions/ 